\title{
Polarization characteristics of photonic quantum ring laser with three-dimensional whispering gallery resonances
}

D. K. Kim, S.-J. An, E. G. Lee, and O'Dae Kwon

Citation: Journal of Applied Physics 102, 053104 (2007); doi: 10.1063/1.2777121

View online: http://dx.doi.org/10.1063/1.2777121

View Table of Contents: http://scitation.aip.org/content/aip/journal/jap/102/5?ver=pdfcov

Published by the AIP Publishing

\section{Articles you may be interested in}

Hybrid whispering gallery mode/plasmonic chain ring resonators for biosensing

Appl. Phys. Lett. 105, 231107 (2014); 10.1063/1.4903876

Three-dimensional whispering gallery modes in InGaAs nanoneedle lasers on silicon

Appl. Phys. Lett. 105, 111105 (2014); 10.1063/1.4895920

Laser oscillations of whispering gallery modes in thiophene/phenylene co-oligomer microrings

Appl. Phys. Lett. 91, 021104 (2007); 10.1063/1.2755925

Spectral analysis of a three-dimensional photonic quantum ring laser with a square microcavity

J. Appl. Phys. 99, 033102 (2006); 10.1063/1.2168236

Evanescent and propagating wave characteristics of the photonic quantum ring laser

Appl. Phys. Lett. 79, 1593 (2001); 10.1063/1.1402655

\section{MIT LINCOLN} LABORATORY CAREERS

Discover the satisfaction of innovation and service to the nation
- Space Control

- Air \& Missile Defense

- Communications Systems \& Cyber Security

- Intelligence, Surveillance and

Reconnaissance Systems
- Advanced

Electronics

- Tactical Systems

- Homeland

Protection

- Air Traffic Control

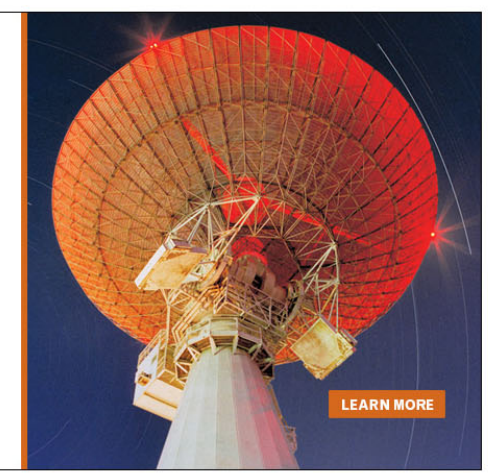




\title{
Polarization characteristics of photonic quantum ring laser with three-dimensional whispering gallery resonances
}

\author{
D. K. Kim, S.-J. An, E. G. Lee, and O'Dae Kwon ${ }^{\text {a) }}$ \\ Department of Electronic and Electrical Engineering, Pohang University of Science and Technology, \\ Pohang, 790-784, Republic of Korea
}

(Received 3 May 2007; accepted 17 July 2007; published online 10 September 2007)

\begin{abstract}
We report polarization characteristics of light from a photonic quantum ring $(\mathrm{PQR})$ laser of three-dimensional (3D) whispering gallery modes. The 3D polarization states of light from the PQR laser were studied with a home-built solid-angle scanner capable of detecting the spatial intensity distribution in a wide range of solid angles. The analysis shows that the dominant polarization vectors of the PQR laser emitting in the surface normal direction lie always tangential to the PQR perimeter, which means there is a strong carrier-photon coupling. (C) 2007 American Institute of
\end{abstract} Physics. [DOI: 10.1063/1.2777121]

\section{INTRODUCTION}

In photonic integrated circuits for high-density optical interconnects and optical signal processing applications, the laser diodes with low threshold, small volume, and high $Q$ factor are essential. For those requirements, microcavity lasers such as microdisk, ${ }^{1-5}$ micropillar, ${ }^{6}$ and microsphere ${ }^{7,8}$ have, until recently, attracted great attention. In particular, thumbtack-type microdisk lasers were intensively studied for low threshold current and high $Q$ factor from the twodimensional (2D) whispering gallery (WG) mode, ${ }^{1,2}$ while the microdisk diodes, shaped like a thumbtack, rather suffer from the structural fragility. Although alternative microcylinder structures ${ }^{9,10}$ have been investigated, the optical confinement was not improved and the threshold current was higher than that of microdisk lasers.

The photonic quantum ring (PQR) laser has now appeared as an attractive candidate for high-density laser chip development, thanks to their unique characteristics such as microampere to nanoampere ultralow threshold currents and $\sqrt{T}$-dependent spectral shifts. ${ }^{11}$ The resonance of the PQR laser results in three-dimensional (3D) WG modes of helical standing waves, ${ }^{12-14}$ which is surface-normal dominant, in contrast to the in-plane 2D WG mode. This 3D WG resonance can also generate angle-dependent emissions as previously reported. ${ }^{13}$ In the present study, we report a 3D polarization-resolved emission analysis of the $\mathrm{PQR}$ from the data taken with a home-built solid angle scanner (SAS) setup and offer a thorough 3D view of the polarization behaviors.

\section{DEVICE STRUCTRUE AND SPECTRAL PROPERTIES}

Cylindrical $\mathrm{PQR}$ structures with the sidewall roughness ${ }^{15}$ being less than $0.7 \mathrm{~nm}$ of about $5 \mu \mathrm{m}$ height etched by chemically assisted ion-beam etching (CAIBE) were fabricated from wafers consisting of an active quantum well region between $n$ - and $p$-type GaAs/AlAs distributed

\footnotetext{
${ }^{a)}$ Author to whom correspondence should be addressed. Electronic mail: odkwon@postech.ac.kr
}

Bragg reflector (DBR) mirrors grown by metal organic vapor-phase epitaxy [Fig. 1(a)]. The active region of one- $\lambda_{0}$ thickness for vertical resonance at $850 \mathrm{~nm}$ wavelength has three $80 \AA$ GaAs quantum wells and two $\mathrm{Al}_{0,3} \mathrm{Ga}_{0.7} \mathrm{As}$ barriers and spacers. Details of fabrication steps such as planarization by polyimide and electrode evaporation are described elsewhere. ${ }^{16}$

Figure 1(b) is a charge coupled device (CCD) image from 3D WG modes of helical standing waves. The spectra from the PQR emission of diameter $20 \mu \mathrm{m}$, collected at polar angles of $\theta=10^{\circ}, 20^{\circ}$, and $30^{\circ}$ in spherical coordinates with an injection current of $3 \mathrm{~mA}$ with the spectrometer resolution of $0.08 \mathrm{~nm}$, are shown in Fig. 1(c). Each spectrum at different polar angles $\theta$ shows an envelope of about $6 \mathrm{~nm}$ span containing several discrete modes. It should be noted that as $\theta$ increases, the envelope is blueshifted successively
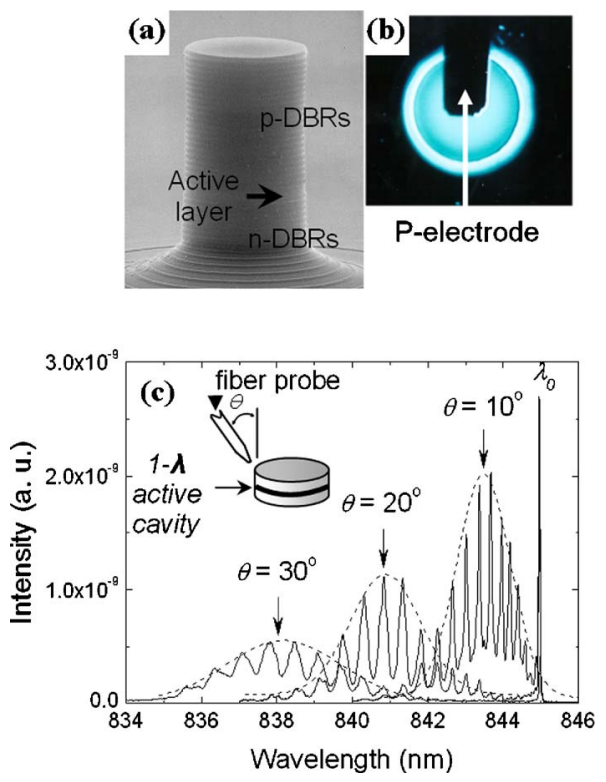

FIG. 1. (Color online) (a) SEM image of a PQR mesa, (b) CCD image of the emission of the PQR laser, which has a diameter of $15 \mu \mathrm{m}$, showing a bright peripheral ring $(I=10 \mu \mathrm{A})$, and (c) PQR spectra measured at polar angles $10^{\circ}, 20^{\circ}$, and $30^{\circ}$, respectively. We note that, when $\theta=0^{\circ}$, the PQR spectrum shows conspicuously sharp vertical line indicated as $\lambda_{0}$. 


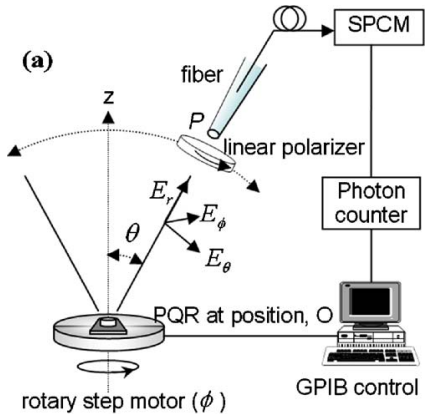

(b)

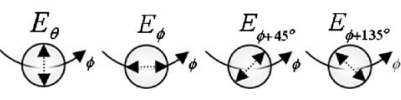

FIG. 2. (Color online) Schematic diagram of solid-angle scanner (SAS): The linear polarizer sweeps the polar angle $\theta$ as shown in (a), while the polarizer orientations are indicated by the dotted arrows in (b).

while the discrete submodes within different envelopes remain the same regardless of $\theta \cdot{ }^{13,14}$ These polar angledependent multichromatic emission characteristics, observed over the regime of a $70^{\circ}$ polar angle, were reported before. ${ }^{17}$

\section{EXPERIMENTAL RESULTS AND DISCUSSION}

We made a SAS (Refs. 18 and 19) to measure the polarization-resolved field intensity distributions of spectrally integrated $\mathrm{PQR}$ optical field emissions. Figure 2(a) shows a schematic diagram of the SAS, which consists of a set of photon detectors and rotary stepmotors for scanning a fiber probe. When the $\mathrm{PQR}$ laser is placed to the origin of the spherical coordinate, a fiber probe with a polarizer (extinction ratio $=0.04 \%$ ), connected to a single photon-counting module (SPCM) (EG\&G) for delicate light detection, scans the north hemisphere from $0^{\circ}$ to $70^{\circ}$ in the polar $(\theta)$ direction and from $0^{\circ}$ to $360^{\circ}$ in the azimuthal $(\phi)$ direction, respectively. The angular resolution is typically $3^{\circ}$ and $2^{\circ}$ for the $\theta$ and $\phi$ directions, respectively. The polarization fields from the PQR are measured with the SPCM which makes a pulse per photon detection event. The number of photon pulses is counted by a photon counter (Stanford Research SR-400). Then, by rotating the linear polarizer, the polarizationresolved light intensity versus polar angle is collected at room temperature. We further note that, as shown in Fig. 1 (c), the total number of photons counted at an angle $\theta$ is the sum of light from all multichromatic modes of different wavelengths.

The orthogonal field components $E_{\theta}$ and $E_{\phi}$ of an optical wave $\left(\bar{E}=E_{\theta} \hat{\theta}+E_{\phi} \hat{\phi}\right)$, coupled to the fiber probe through a linear polarizer at position $P$, contain multichromatic submode frequencies and wave vectors within an envelope. The intensities of two orthogonally polarized electric field components can then be expressed as the time-averaged values of the complex amplitude of each electric field $\left(I_{\theta}=\left\langle E_{\theta}^{2}\right\rangle_{T}\right.$ and $\left.I_{\phi}=\left\langle E_{\phi}^{2}\right\rangle_{T}\right) . I_{\theta}$ and $I_{\phi}$ correspond to the number of average photons passing through the linear polarizer whose transmission axis is fixed parallel to the $\theta$ and $\phi$ directions, respectively. The fiber probe waits for $1 \mathrm{~s}$ at each position $(r, \theta, \phi)$ before measurements are made.
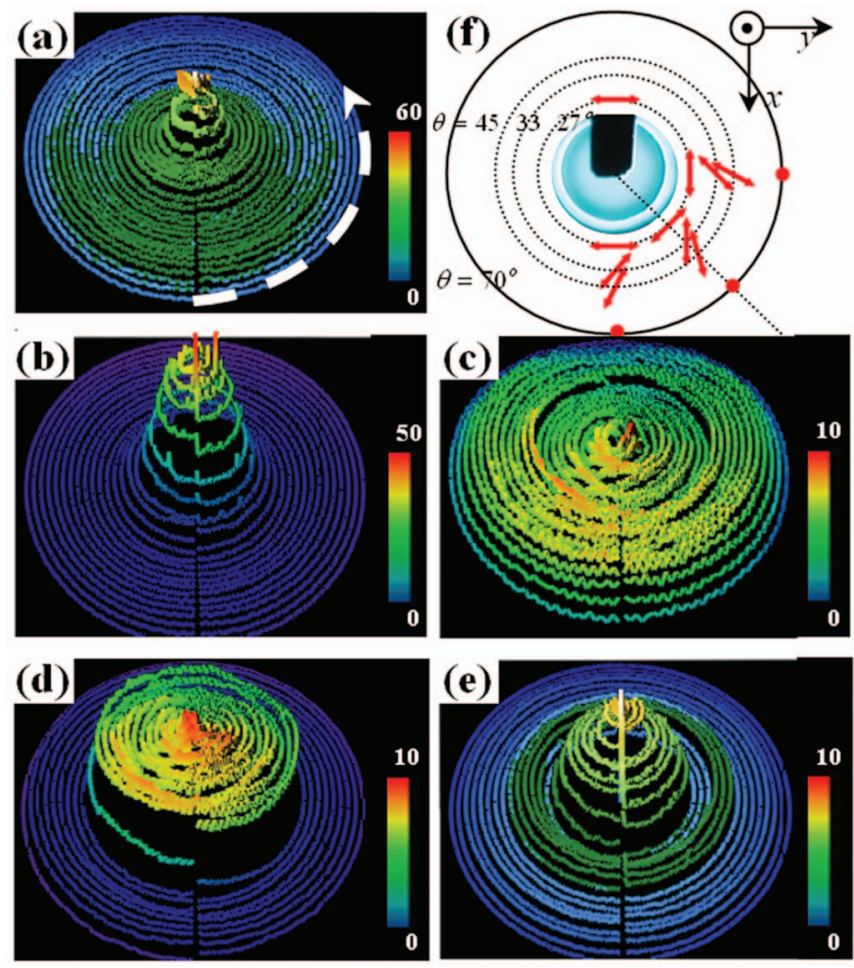

FIG. 3. (Color) Plots of intensity on 2D surface $(x, y)$ from 3D spatial intensity distribution data (a) without polarizer, with (b) $\phi$, (c) $\theta$, (d) $\phi$ $+45^{\circ}$, (e) $\phi+135^{\circ}$ directional polarizers, and (f) summary of polarization states according to polar angle. Three red dots represent polarization states of unit $\theta$ vectors.

Figure 3(a) plots the intensity on a 2D surface $(x, y)$ converted from $3 \mathrm{D}$ spatial intensity distribution data on a north-hemisphere surface ( $r=$ const, $0^{\circ} \leqslant \theta \leqslant 70^{\circ}, 0^{\circ} \leqslant \phi$ $\leqslant 360^{\circ}$ ) without the polarizer for the $\mathrm{PQR}$ of diameter $8 \mu \mathrm{m}$ with $150 \mu \mathrm{A}$ injection current of $10 \mathrm{kHz}$ pulse (50\% duty). Mapping is defined by $\theta=\pi \sqrt{x^{2}+y^{2}}, \phi=\arg (x+y i) .{ }^{19}$ The dotted arrow in Fig. 3(a) represents the scanning direction of increasing $\phi$, and a specific vertical direction of $\theta=0^{\circ}$ corresponds to the center $(x=0, y=0)$ of circular $2 \mathrm{D}$ plot. Figures 3(b)-3(e) display the intensities when the transmission axis of the linear polarizer is set parallel to the $\phi, \theta, \phi+45^{\circ}$, and $\phi+135^{\circ}$ directions [as illustrated in the four diagrams of Fig. 2(b)], respectively, under the same bias conditions. We note that our data taken with the SPCM suffers photon counting errors. The maximum count fluctuations are $2.4 \%$ in Fig. 3(a), 25\% in Fig. 3(b), 3\% in Fig. 3(c), 30\% in Fig. 3(d), and $21 \%$ in Fig. 3(e), and the spatial discontinuities are not real since they are nonphysically generated simply due to a 3D representation of 2D data for visual assistance. In this experiment, the range of polar angle to be scanned was limited by $0^{\circ} \leqslant \theta \leqslant 70^{\circ}$ due to the $\mathrm{PQR}$ device structure such as protruding metal electrodes, package frame, and bulky fiber probes.

From the intensity comparison between Figs. 3(a) and 3 (b) we know that the polarization vectors of light propagating along the nearly perpendicular direction from the PQR laser lie tangential to the quantum ring perimeter, that is, parallel to the $\phi$ vector. Also from Figs. 3(a) and 3(c), the $\theta$ vector polarization almost dominates near the surface parallel $\left(\theta \sim 70^{\circ}\right)$, contrary to the case of $2 \mathrm{D}$ microdisk lasers 
revealing the TE polarization vector ${ }^{1,20}$ where the electric field is dominantly parallel to the disk plane. This polarization phenomenon reflects the nontrivial aspects of the 3D WG modes of the PQR helix waves. Moreover, the observed nonsymmetry between $\phi+45^{\circ}$ and $\phi+135^{\circ}$ intensity data, as shown in Figs. 3(d) and 3(e), may imply a distinctively unbalanced presence of clockwise and counterclockwise helix modes. This feature may have to be analyzed in further detail by studying the device's orbital angular momenta. ${ }^{21,22}$ The dominant $\phi$ polarization state of $\mathrm{PQR}$ (parallel to the quantum well gain plane) at low polar angles reflects the $\phi$ polarization enhanced due to larger optical gains for TE modes. However, the dominant $\theta$ polarization perpendicular to the gain planes observed at high polar angles means that higher Fresnel reflection coefficient for the $\theta$ polarization state gives rise to higher optical mode confinements, as well as reflectivity, at the edge of the device.

The intensity profile of Fig. 3(a) is a superposition of Figs. 3(b) and 3(c), that is, $I=I_{\theta}+I_{\phi}$. Additionally, considering Figs. 3(d) and 3(e) for the state of polarization within an intermediate region of $27^{\circ} \leqslant \theta \leqslant 70^{\circ}$, the polarization vectors of light from the $\mathrm{PQR}$ laser are gradually reoriented from the $\phi$ vector to the $\theta$ as the polar angle $\theta$ increases; all of the results are summarized in Fig. 3(f). For more details on the degree of polarization, we may use a polarization rate defined as $V=\left(I_{\phi}-I_{\theta}\right) /\left(I_{\phi}+I_{\theta}\right)$, and we get values of $86 \%$ and $-5 \%$ for the polar angles of $10^{\circ}$ and $70^{\circ}$, respectively. This means that although the $\mathrm{PQR}$ polarizations are elliptical in general, their major components are $\phi$ polarization vector near $\theta=0^{\circ}$ and $\theta$ polarization vector near $\theta=90^{\circ}$, respectively. The rectangular metal electrode shown in Figs. 1(b) and 3(f) slightly distorts the circular symmetry of the 3D $\mathrm{PQR}$ emission, as observed in the non-symmetric intensity pattern between $\phi=0^{\circ}$ and $\phi=180^{\circ}$ regions, shown in Fig. 3(a).

Once we understand the above behaviors of the PQR laser's polarization vectors, the polarization can be predictably controlled by proper $\mathrm{PQR}$ designs, whereas the difficulty of polarization controls are well known for the case of the vertical cavity surface emitting lasers. ${ }^{23,24}$ On the other hand, to compare the present 3D WG polarization properties with the 2D microdisk lasers, one has to be very careful: The 3D WG components corresponding to the 2D polarization will be $3 \mathrm{D}$ polarization data at $\theta=90^{\circ}$, while the present $3 \mathrm{D}$ data taken at $\theta=70^{\circ}$ are already suffering from very low intensities as shown in Fig. 3.

\section{SUMMARY}

We have constructed an SAS experimental setup and analyzed the polarization characteristics of the $\mathrm{PQR}$ laser having 3D WG resonance properties with the polar angle dependence up to $\theta=70^{\circ}$. The analysis showed that the surface normal components are lying tangential to the PQR perimeter, ${ }^{25}$ which means that a tendency of strong carrierphoton coupling is realized when the recombinant carrier distributions become quantum-wire-like momentarily. ${ }^{26}$ Also, it was observed that as the polar angle increased from $0^{\circ}$ to $70^{\circ}$, the polarization vector of light from the PQR laser was systematically reoriented from the $\phi$ vector direction tangential to the PQR perimeter, nearly to the $\theta$ vector direction perpendicular to the equator.

\section{ACKNOWLEDGMENTS}

This research was sponsored by the National Research Lab., KOSEF, Samsung Co., and BK21.

${ }^{1}$ S. L. McCall, A. F. J. Levi, R. E. Slusher, S. J. Pearton, and R. A. Logan, Appl. Phys. Lett. 60, 289 (1992).

${ }^{2}$ A. F. J. Levi, R. E. Slusher, S. L. McCall, T. S. J. Pearton, and W. S. Hobson, Appl. Phys. Lett. 62, 2021 (1993).

${ }^{3}$ M. K. Chin, D. Y. Chu, and S. T. Ho, J. Appl. Phys. 75, 3302 (1994).

${ }^{4}$ C. Gmachl, F. Capasso, E. E. Narimanov, J. U. Nöckel, A. D. Stone, J. Faist, D. L. Sivco, and A. Y. Cho, Science 280, 1556 (1998).

${ }^{5}$ P. Koonath, T. Indukuri, and B. Jalali, Appl. Phys. Lett. 86, 091102 (2005).

${ }^{6}$ G. D. Chern, H. E. Tureci, A. D. Stone, R. K. Chang, M. Kneis, and N. M. Johnson, Appl. Phys. Lett. 83, 1710 (2003).

${ }^{7}$ X. Fan, A. Doran, and H. Wang, Appl. Phys. Lett. 73, 3190 (1998).

${ }^{8}$ M. Cai, O. Painter, and K. J. Vahala, Opt. Lett. 25, 1430 (2000).

${ }^{9}$ S. M. K. Thiyagarajan, D. A. Cohen, A. F. J. Levi, S. Ryu, R. Li, and P. D. Dapkus, Electron. Lett. 35, 1252 (1999).

${ }^{10}$ M. Fujita, R. Ushigome, T. Baba, A. Matsutani, F. Koyama, and K. Iga, Jpn. J. Appl. Phys., Part 1 40, 5338 (2001).

${ }^{11}$ J. C. Ahn, K. S. Kwak, B. H. Park, H. Y. Kang, J. Y. Kim, and O’Dae Kwon, Phys. Rev. Lett. 82, 536 (1999).

${ }^{12}$ B. H. Park, J. Bae, M. J. Kim, and O'Dae Kwon, Appl. Phys. Lett. 81, 580 (2002).

${ }^{13}$ J. W. Bae, J. W. Lee, O’Dae Kwon, and V. G. Minogin, Opt. Lett. 28, 1861 (2003).

${ }^{14}$ J. W. Bae, J. W. Lee, D. K. Kim, J. Y. Kim, and O'Dae Kwon, Appl. Opt. 42, 5508 (2003)

${ }^{15}$ M. J. Kim, D. K. Kim, S. E. Lee, and O’Dae Kwon, J. Appl. Phys. 96, 4742 (2004)

${ }^{16}$ J. Y. Kim, K. S. Kwak, J. S. Kim, B. K. Kang, and O'Dae Kwon, J. Vac. Sci. Technol. B 19, 1334 (2001).

${ }^{17}$ J. C. Ahn, H. Y. Kang, and O’Dae Kwon, Proc. SPIE 3283241 (1998).

${ }^{18}$ T. D. Lee, P. H. Cheng, J. S. Pan, R. S. Tsai, Y. Lai, and K. Tai, Appl. Phys. Lett. 72, 2223 (1998).

${ }^{19}$ D. J. Shin, S. H. Kim, J. K. Hwang, H. Y. Ryu, H. G. Park, D. S. Song, and Y. H. Lee, IEEE J. Quantum Electron. 38, 857 (2002).

${ }^{20}$ N. C. Frateschi, A. P. Kanjamala, A. F. J. Levi, and T. Tanbun-Ek, Appl. Phys. Lett. 66, 1859 (1995).

${ }^{21}$ L. Allen, M. W. Beijersbergen, R. J. C. Spreeuw, and J. P. Woerdman, Phys. Rev. A 45, 8185 (1992).

${ }^{22}$ P. Z. Dashti, F. Alhassen, and Henry P. Lee, Phys. Rev. Lett. 96, 043604 (2006).

${ }^{23}$ K. Iga, IEEE J. Sel. Top. Quantum Electron. 6, 1201 (2000).

${ }^{24}$ D. L. Boiko, G. Guerrero, and E. Kapon, Opt. Express 12, 2597 (2004).

${ }^{25}$ D. K. Kim, E. G. Lee, and O'Dae Kwon, Electron. Lett. 43, 188 (2007).

${ }^{26}$ O'Dae Kwon et al., Appl. Phys. Lett. 89, 011108 (2006). 